\title{
Numerical analysis of blood flow in human abdominal aorta
}

\author{
M. Podyma ${ }^{1}$, I. Zbicinski ${ }^{1}$, J. Walecki ${ }^{2}$, M. L. Nowicki ${ }^{2}$, \\ P. Andziak ${ }^{3}$, P. Makowski ${ }^{4}$ \& L. Stefanczyk ${ }^{5}$ \\ ${ }^{1}$ Faculty of Process and Environmental Engineering, \\ Technical University of Lodz, Poland \\ ${ }^{2}$ Department of Radiology, Postgraduate Medical Center, Poland \\ ${ }^{3}$ Surgical Clinic, Central Clinical Hospital MSWiA, Poland \\ ${ }^{4}$ Faculty of Electrical and Electronic Engineering, \\ Technical University of Lodz, Poland \\ ${ }^{5}$ Department of Radiology and Diagnostic Imaging, \\ Medical University of Lodz, Poland
}

\begin{abstract}
A CFD model of flow hemodynamics in abdominal aortic aneurysm to estimate the effect of four different flow conditions on velocity and pressure profiles was developed and verified. The reconstruction of blood flow domain geometry was performed on the basis of CT examination. Our own software was used for CT image segmentation and real blood flow domain geometry reconstruction. Data necessary for determination of initial and boundary conditions as well as for verification of developed CFD model were collected from USG-Doppler examinations at 4 characteristic areas of the abdominal aorta. The result of these calculations proved that, irrespective of the applied boundary conditions and flow type, velocity profiles in all of the analysed models were similar. The comparison of theoretical and experimental results showed a significant difference in the absolute values of both velocities and heart-work period length. The reasons for the discrepancies were analyzed indicating both numerical factors and the character and accuracy of the USG examination.

Keywords: numerical simulation, verification of CFD model, hemodynamics, segmentation, CT, USG-Doppler.
\end{abstract}




\section{Introduction}

Human body seen from chemical engineering perspective is a reactor, in which momentum, heat and mass transfer processes accompanied with chemical and biochemical reactions take place. Recent development of experimental techniques and theoretical methods allowed us to observe many distinctive phenomena, particularly in the area of blood flow dynamics. One of developed methods, the CFD technique, enabled analysis of flow hemodynamics in the blood vessel. The CFD models can be used to calculate distributions of tensile stresses expanding the walls of vascular segments and hydraulic resistance. Information on stress distributions, i.e. on hemodynamic forces that accompany blood flow, is very important to indicate areas that are threatened with a vascular wall rupture and helpful in prediction of the rupture time.

There are two simultaneously occurring factors that provoke aneurysm; biochemical ones that cause local lesions of vascular walls, and hemodynamic which lead to dilation of the weakened aorta segment. Hemodynamic factors that have an influence on aneurysm formation include, among the others, disturbance of laminar blood flow, eddies, increased flow rate and first of all raised blood pressure. Hence, attempts of predicting the areas that are specially vulnerable to the above mentioned factors must be based on the real system geometry that reflects anatomic details specific of a given patient, such as local arteriostenosis, presence of clots, etc.

None of the currently applied non-invasive diagnostic methods provides information on shear stress that appears within aortic walls which leads to the aorta rupture. A strategic aim of this project is to develop a mathematical model to determine the growth rate of aortic aneurysm on the basis of flow hemodynamics and mechanical properties of vascular walls.

In literature, there are numerous references on the flow in blood vessels; but there are no papers that refer at the same time to the flow hemodynamics and the behavior of vascular walls. Only such approach can enable specification of the conditions in which aortic aneurysm will form and grow.

The basic target of this work was to construct and verify a CFD model of flow hemodynamics in aortic aneurysm and to assess the effect of initial and boundary conditions on quality of calculations of blood flow related to local narrowing of aorta lumen.

\section{Medical data}

Patients suffering from aneurysm have been selected and monitored more frequently than in a standard medical procedure. A criterion for including patients into the program was the growth rate of aneurysm diameter. Each patient was subjected to clinical examinations (CT, USG-Doppler, angiography) that enabled determination of hemodynamic parameters of blood vessels (linear dimensions, aneurysm volume increment, the amplitude of vascular wall oscillations, etc.) and development of kinetic equations which control the aneurysm growth rate. 
The reconstruction of blood flow domain geometry was performed on the basis of CT examination. Data necessary for determination of initial and boundary conditions as well as for verification of developed CFD model were collected from USG-Doppler examinations at 4 characteristic areas of abdominal aorta: below renal aorta (inlet) - A, aneurysm neck $-\mathrm{B}$, distal part $-\mathrm{C}$, iliac arteries - D1, D2 (see Fig. 1a).

\section{Flow geometry}

In order to reconstruct the real geometry on the basis of computer tomograms (CT) we used our own software for 2D image processing, extraction of 3D geometry based on the series of cross sections of the reconstructed organ (aorta) and numerical mesh generation.

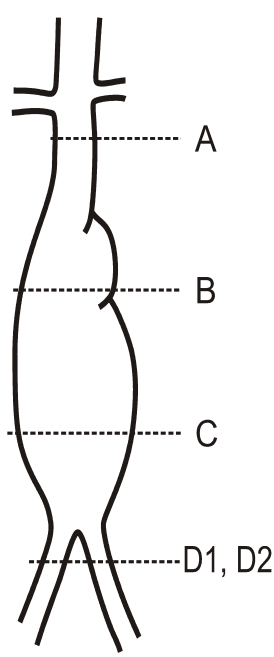

a)

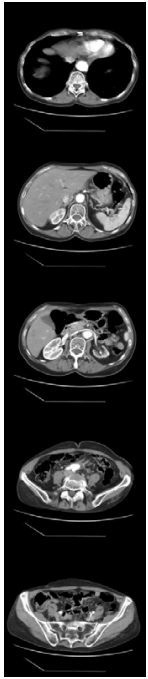

b)

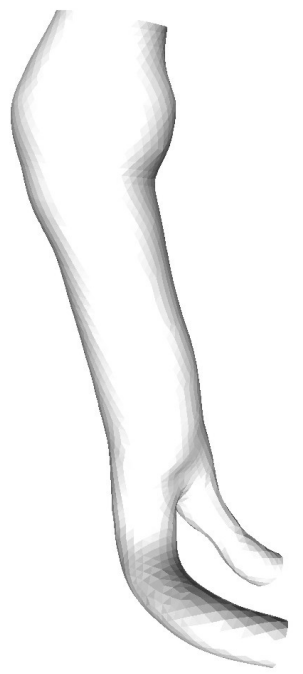

c)

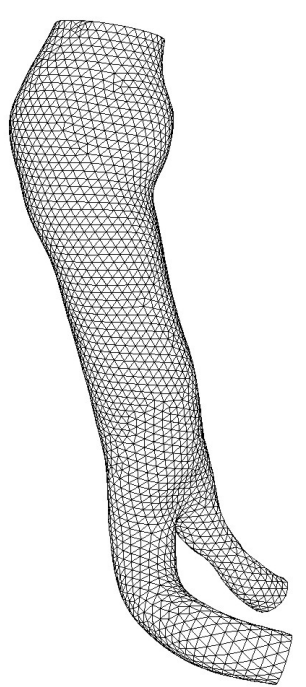

d)

Figure 1: Abdominal artery geometry: a) USG-Doppler measurement points, b) CT scan data, c) 3D geometry of blood flow domain (STL), d) $3 \mathrm{D}$ volume mesh (tetrahedral elements).

The expected functionality of the segmentation is a transformation from a series of planar CT images into a 3D structure, representing the lateral surface of blood vessels. The obtained structure indirectly defines the geometrical boundary conditions for CFD simulations. The segmentation method used in this paper has been presented in [1] and modified due to a larger vessel diameter. It allowed us to determine the geometry of the vessel using approximation of the vessel shape by a series of ellipses located in planes perpendicular to the vessel axis.

The method has been initially developed for segmentation of the coronary arteries, significantly smaller in diameter than the aorta with aneurysm described 
here. Therefore, the main adaptation, which has been introduced in the frame of this work, was a modification of the assumed shape of the vessel cross-section. Instead of an ellipse with 2 radii [1] we introduced an asymmetrical ellipse with 4 radii. We have also implemented automatic fitting of the ellipse to the image data. In an iterative process we are able to change 7 parameters (position, radii, rotation angle), which results in rigid deformation of the ellipse. The method was efficient for small and middle diameter of the vessel. For large part of the aorta, automatic segmentation often failed and manual adjustment of parameters was required. The main advantage of the segmentation with an approximated, 4-radii elliptical shape is immunity to image artifacts resulting in a smooth surface of the vessel.

Flow geometry obtained in this way was described in a universal STL format (Stereo lithography) and imported into a Gambit 2 numerical preprocessor (Fluent INC). On the basis of STL geometry, a three-dimensional tetrahedral volume mesh of flow domain was generated. Preliminary calculations were performed to get the mesh independent solution (50,000 cells and double density about 100,000 cells). We have found that both meshes delivered results with similar accuracy of calculations, so finally the lower density mesh (50,000 cells) was selected.

\section{CFD model}

In numerical simulations a commercial CFD package Fluent 6 was used. A numerical grid representing flow geometry was generated on the basis of CT examinations for a selected patient. Calculations were performed in unsteadystate conditions.

\subsection{Boundary conditions}

Blood flow velocities in selected areas (Fig. 1a) of the abdominal aorta were taken from a USG-Doppler examination. After USG velocity spectrum (Fig. 2) analysis, real transient blood flow velocity profiles were extracted and described by the Fourier series, eqn. (1).

$$
u(t)=\frac{a_{0}}{2}+\sum_{n=1}^{\infty}\left(a_{n} \cos (n \omega t)+b_{n} \sin (n \omega t)\right)
$$

where $a_{n}$ and $b_{n}$ are Fourier coefficients. Twelve Fourier terms were considered to obtain satisfactory approximation of the velocity profile.

Data taken at A position were used to determine transient velocity profile at the inlet to the flow domain (inlet boundary condition). Data from B, C, D1, D2 were used to verify CDF calculation results.

\subsection{CFD model parameters}

Blood flow in the cardiovascular system caused by cardiac contractions has pulsating character and for big vessels is usually in laminar and transient range. To analyze effect of type of the flow and pressure boundary conditions on 
accuracy of calculations four independent hemodynamics models developed for turbulence an laminar flow and constant and transient pressure at the outlet of the aorta. The list of analyzed models is given below.

1. Turbulent flow + constant pressure at the outlet (TCP)

2. Turbulent flow + transient pressure at the outlet (TTP)

3. Laminar flow + constant pressure at the outlet (LCP)

4. Laminar flow + transient pressure at the outlet (LTP)
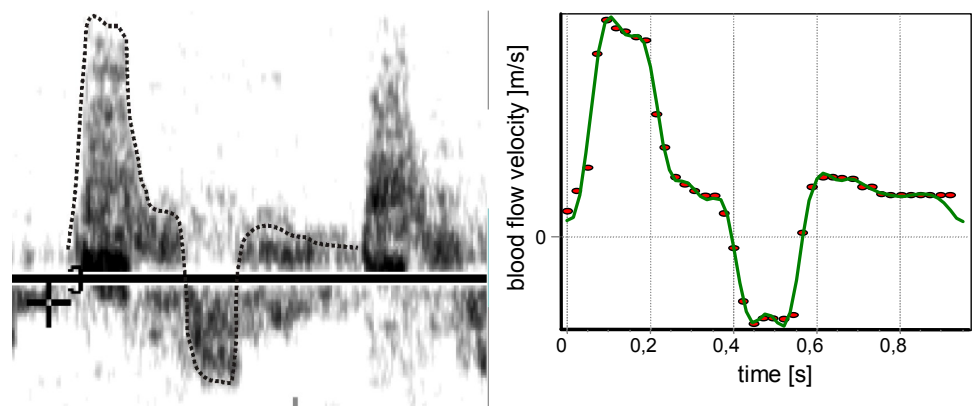

Figure 2: $\quad$ Analysis of USG-Doppler data and Fourier series fit.

In all calculations for turbulent flow Large Eddy Simulation (LES) model was applied [2]. Conceptually, the large eddy simulation (LES) is a compromise between DNS (Direct Numerical Simulation) having large computational cost and the RANS (Reynolds-averaged Navier-Stokes) approach.

Arterial blood pressure in big vessels of a healthy man ranges from about $9.3 \mathrm{kPa}(70 \mathrm{~mm} \mathrm{Hg})$ to around $16 \mathrm{kPa}(120 \mathrm{~mm} \mathrm{Hg})$. For both models (laminar and LES), calculations were performed at constant pressure $(11.6 \mathrm{kPa})$ and changing in time pressure profile. A transient profile of outlet pressure was generated on the basis of systolic a diastolic pressures of a selected patient. To describe non-Newtonian properties of blood, Quemada's rheological model was applied [3,4]. Blood density was assumed constant and equal to $1040 \mathrm{~kg} / \mathrm{m}^{3}$ [6].

\section{Results}

Selected results of the calculations referring to local changes in blood flow velocity and pressure in one heart-work period are shown in Figs. 3 through 5. Figure 3 shows calculated blood velocity profiles in selected cross sections of the aorta (see Fig. 1) for all models.

Analysis of Fig. 3 indicates that irrespective of the applied boundary conditions and flow type, the velocity profiles in all four models are similar and so subsequent comparative analyses concerning blood flow velocities are based on the most complex model (TTP). Setting of the boundary condition at the inlet in the form of blood velocity profile determines its distribution in the whole flow area with the difference between the inlet and outlet pressure being preserved. 

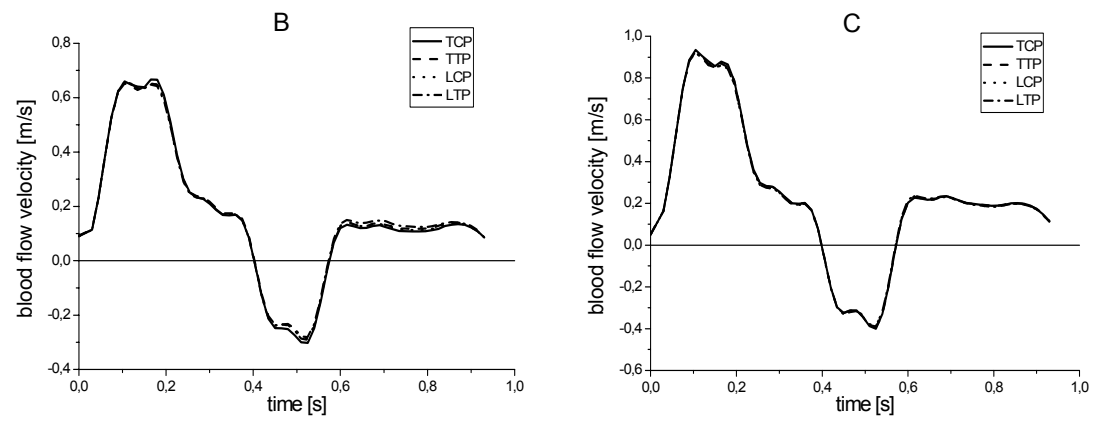

Figure 3: Calculated blood velocity profiles for all models in selected cross sections of the aorta (see Fig. 1).

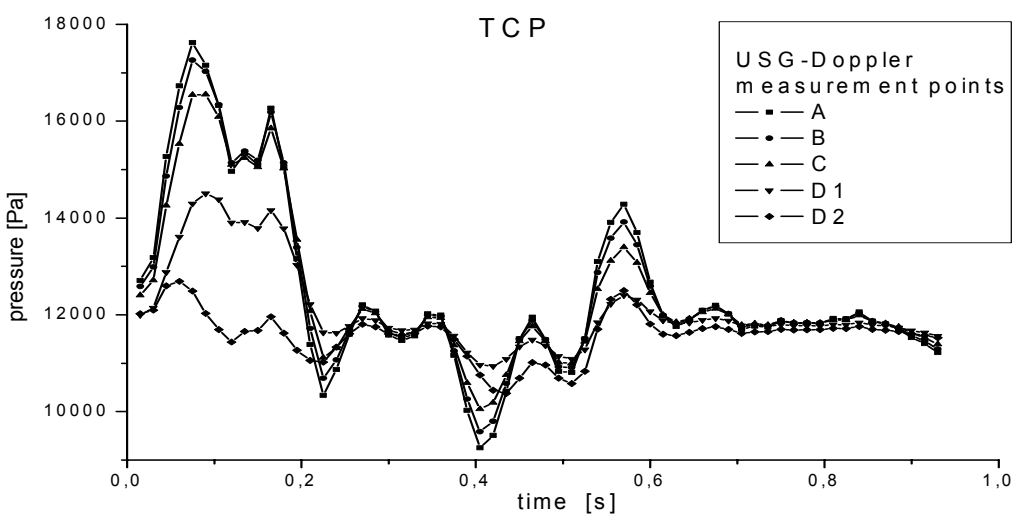

Figure 4: Calculated pressure profiles for models TCP.

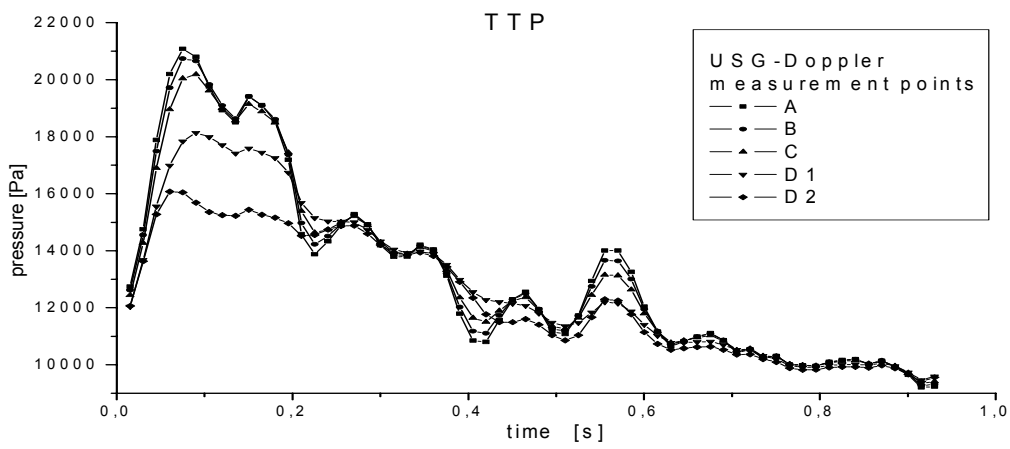

Figure 5: Calculated pressure profiles for models TTP. 
A change in the outlet pressure causes only a change in the operation pressure in the artery with constant pressure difference between the analyzed cross section of the aorta (Fig. 4 and 5). In the models with constant outlet pressure, a gradual flattening of the pressure profile until reaching a constant level controlled by the boundary condition, can be observed (Fig. 4). It is worth noting that such a boundary condition may lead to underestimation of pressure in the artery, and as a result to errors in wall stress determination. In view of this, despite correct transient velocity profiles in the models with constant pressure one should apply transient pressure boundary conditions (Fig. 5).

Comparison of the calculations shows also that when the turbulence model (LES) is taken into account in the calculations, this has no effect of blood flow velocity profiles in the aorta which means that real flow in the system is close to laminar one $[5,7]$.

The main aim of this study, however, was to compare results of theoretical calculations of blood flow velocity profiles with USG experimental data. The USG velocity profiles taken at position A were used as the initial boundary condition for each of four models. Velocity profiles obtained at cross sections B, C, D1, D2 (Fig. 1) were used to verify CDF calculation results.

B

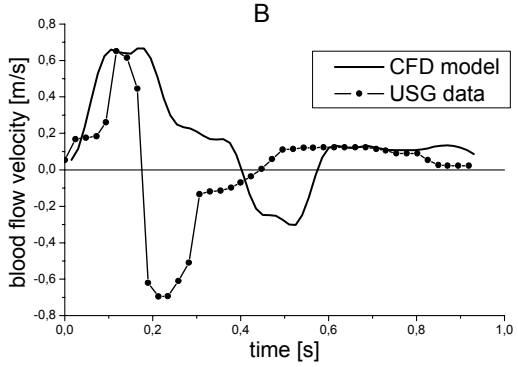

D1

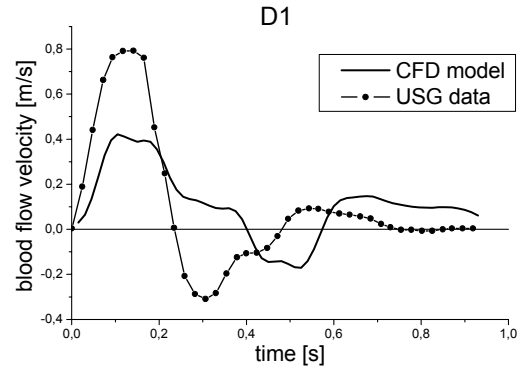

C

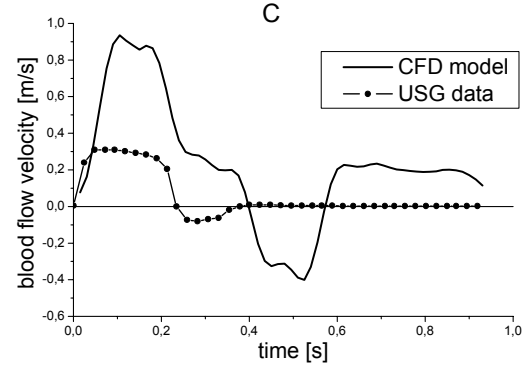

D2

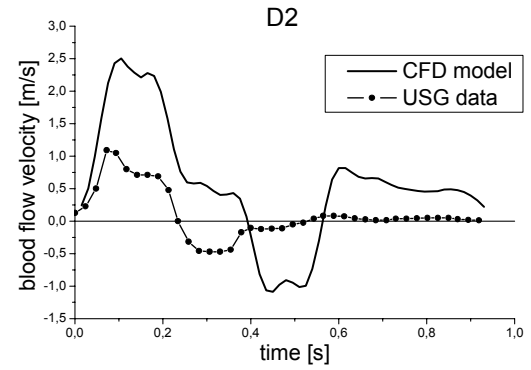

Figure 6: Blood flow velocity profiles at four aorta positions (see Fig. 1) comparison of calculations results with USG-Doppler data.

Comparison of theoretical and experimental results shows significant difference in the absolute values of both velocities and heart-work period length (see Fig. 6). Reasons of the discrepancies can be both numerical (e.g. the 
pulsations of aorta walls are not included into the calculations) and these related to the character and accuracy of USG measurements.

The abdominal aorta is an organ difficult to access which seriously hampers proper USG examinations. A high correction angle (about $60^{\circ}$ ) causes that even slight deviations (of the order of $3^{\circ}$ ) result in velocity measuring errors reaching even $30 \%$ (we use convex USG probe). From the technical point of view, the USG measurement cannot be considered precise because of difficulties in setting the correction angle, USG probe position and artifacts (noise) arising during the examination.

Additionally, during a USG test lasting for several minutes, a patient's heart rhythm changes due to stress, breathing fluctuations, changes of body position, arrhythmia, etc. These disorders cause that duration of diastolic phase changes [8] which can explain the phase shift shown in the diagrams in Fig. 6.

As it is not possible to fully verify the calculations, results obtained using the CFD models of blood flow in the abdominal aorta have a qualitative rather than quantitative character.

\section{Conclusions}

1. Despite of correct transient velocity profiles obtained in all models considered, in aortic blood flow calculations the transient pressure boundary conditions should be applied because of a possible underestimation of arterial pressure and as a consequence errors in the determination of wall stress.

2. Due to inaccuracies of USG measurements and possible numerical uncertainties, no full verification of the CFD calculations of blood flow in the aorta was possible. As a result, the CFD modeling of hemodynamics in the abdominal aorta has a more qualitative than quantitative character.

\section{Acknowledgement}

Financial support from the State Committee for Scientific Research (Grant No. 3T09C 033 26) is gratefully acknowledged.

\section{References}

[1] Makowski P., Ringgaard S., Fruend E. T., Pedersen E. M., A Novel Approach to Segmentation of Coronary Arteries in MR Images for Computational Fluid Dynamics (CFD) Simulations, 2004 ISMRM, Kyoto, Japan.

[2] FLUENT 6.1 Documentation, Fluent INC.

[3] Cokelet G. R., The rheology of human blood. Biomechanics, its fundamentals and objectives, Prentice Hall, Engelwood, Cliffs, New York. 1972, 63. 
[4] Quemada D., Rheology of concentrated disperse systems. III. General features of the proposed non-Newtonian model. Comparison with experimental data. Rheol. Acta, 1978, 17, 632.

[5] Leondes C., Biomechanical Systems Techniques and Applications, Vol. IV Biofluid Methods in Vascular and Pulmonary Systems; CRC Press LLC. 2001.

[6] Bebenek B., Flows in cardiovascular system, Cracow. ZGPK (in polish). 1999.

[7] Lieber, B. B., Arterial Macrocirculatory Hemodynamics, The Biomedical Engineering Handbook: Second Edition. Ed. Joseph D. Bronzino. Boca Raton. CRC Press LLC, 2000.

[8] Humes H. D., Kelley's Textbook of Internal Medicine, Lippincott Williams \& Wilkins (LWW), 2000. 